\title{
DETERMINATION OF COINTEGRATING RANK IN FRACTIONAL SYSTEMS ${ }^{\star}$
}

\author{
by \\ Peter M Robinson \\ London School of Economics and Political Science \\ and \\ Yoshihiro Yajima \\ University of Tokyo
}

Contents:

Abstract

1. Introduction

2. Fractional integration and cointegration

3. Testing the homogeneity of fractional difference parameters

4. Determination of fractional cointegrating rank

5. Empirical example

6. Final comments

Appendix: Proofs

References

Tables $1-5$

Discussion Paper

No.EM/01/423

July 2001
The Suntory Centre

Suntory and Toyota International Centres for Economics and Related Disciplines London School of Economics and Political Science Houghton Street London WC2A $2 \mathrm{AE}$ Tel.: 020-7955 6698

The authors are especially grateful for help with data analysis provided by Professor K Nawata and Mr T Okimoto of the University of Tokyo and by $\operatorname{Dr} \mathrm{Y}$ Matsuda of Niigata University. They thank two referees for a careful reading of the paper and helpful comments. They also thank Dr L Giraitis of the London School of Economics for helpful comments and $\mathrm{Dr} S$ Kuriki of the Institute of Statistical Mathematics for advice on the closed testing method. Robinson's research was supported by a Leverhulme Trust Personal Research Professorship and ESRC Grants R000235892 and R000238212. Yajima's research was supported by the Ministry of Education of Japan, reference number 09\#CE2002. 


\begin{abstract}
This paper develops methods of investigating the existence and extent of cointegration in fractionally integrated systems. We focus on stationary series, with some discussion of extension to nonstationarity. The setting is semiparametric, so that modelling is effectively confined to a neighbourhood of frequency zero. We first discuss the definition of fractional cointegration. The initial step of cointegration analysis entails partitioning the vector series into subsets with identical differencing parameters, by means of a sequence of hypopthesis tests. We then estimate cointegrating rank by analysing each subset individually. Two approaches are considered here, both of which are based on the eigenvalues of an estimate of the normalised spectral density matrix at frequency zero. An empirical application to a trivariate series of oil prices is included.
\end{abstract}

Keywords: Fractional cointegration; long memory.

JEL No.: C22

(C) by the authors. All rights reserved. Short sections of text, not to exceed two paragraphs, may be quoted without explicit permission provided that full credit, including (c) notice, is given to the source.

Contact address: Professor Peter M Robinson, Department of Economics, London School of Economics and Political Science, Houghton Street, London WC2A 2AE, e-mail: pm.robinson@lse.ac.uk 


\section{Introduction}

Cointegration analysis has principally been developed theoretically, and applied empirically, in the " $I(1) / I(0)$ " context, in which components of a multivariate empirical series displaying evidence of unit root nonstationarity $(I(1)))$ are examined for the existence of one or more stationary, short memory $(I(0))$ linear relations. We call a scalar time series $u_{t}, t=0, \pm 1, \pm 2, \ldots$, $I(0)$ if it is covariance stationary and has spectral density that is finite and positive at zero frequency; for such a $u_{t}$ series we call the scalar series $v_{t}=\left(u_{1}+\ldots+u_{t}\right) 1(t>0), t=0, \pm 1, \pm 2, \ldots, I(1)$, where $1($.$) is the$ indicator function. Then a $p \times 1$ vector variate $X_{t}$ with $I(1)$ elements has been said to be cointegrated if there exists a linear combination $\beta^{\prime} X_{t}$, the

prime denoting transposition, that is $I(0)$. If there are $r, 1 \leq r \leq p-1$, such relations, with linearly independent coefficients, we can express $X_{t}$ in terms of unobservable components,

$$
X_{t}=A F_{t}+V_{t}, \quad t \geq 1
$$

where $F_{t}$ is a $(p-r) \times 1$ vector of unobservable (not cointegrated) $I(1)$ series, $A$ is a full rank $p \times(p-r)$ matrix, and $V_{t}$ is a $p \times 1$ vector of unobservable $I(0)$ series; $F_{t}$ can be interpreted as a vector of common trends [see e.g., Stock and Watson (1988)]. The integer $r$ is termed the cointegrating rank and the $\beta^{\prime} X_{t}$ are termed cointegrating errors. This basic setup has been extended in various directions, for example to incorporate deterministic trends, but the basic notion of observables with an $I(1)$ component, and of $I(0)$ cointegrating errors, is standard. Many tools of statistical inference have been developed to 
investigate the existence of such cointegration, and widely applied empirically [see e.g. Banerjee et al. (1993), Engle and Granger (1991), Hatanaka (1996), Johansen (1996)], for example in connection with consumption and income data, term structure of interest rates, and purchasing power parity between exchange rates.

The possibility of a long-run stable relationship existing between nonstationary series $X_{t}$ exists irrespective of whether or not the series are $I(1)$, however. Indeed, one can also envisage the possibility of cointegration with $\beta^{\prime} X_{t}$ stationary but not necessarily $I(0)$, while cointegration can also be defined for stationary $X_{t}$. There is considerable interest in identifying structure in multivariate series, and thus a strong case for a flexible approach. This is permitted by the class of $I(d)$ series, with real-valued $d$. For $d<1 / 2$, we say $v_{t}$ is $I(d)$ if $u_{t}=(1-L)^{d} v_{t}$ is $I(0)$, where $L$ is the lag operator and

$$
(1-L)^{d}=\sum_{j=0}^{\infty} \frac{\Gamma(j-d)}{\Gamma(-d) \Gamma(j+1)} L^{j},
$$

where $\Gamma(a)=\int_{0}^{\infty} x^{a-1} e^{-x} d x$ for $a>0$, while for $a=-n, n=0,1, \ldots, \Gamma(a)$ has simple poles wih residues $(-1)^{n} / n$, and for other $a<0 \Gamma(a)$ is obtainable by the recursion $\Gamma(a)=\Gamma(a+1) / a$. Then $v_{t}$ is covariance stationary. For $d \geq$ $1 / 2$, we define a nonstationary $I(d)$ series $v_{t}=(1-L)^{-d}\left\{u_{t} 1(t>0)\right\}$. Clearly this $I(d)$ class nests the $I(0)$ and $I(1)$ series. The parameter $d$, called the fractional differencing parameter, can be said to describe the memory of $v_{t}$. In fact, while the early paper of Engle and Granger (1987) focussed principally on cointegration in the " $I(1) / I(0)$ " context, it included a definition which applies to $I(d)$ series (though its $I(d)$ class is much narrower than ours): $X_{t}$ can be said to be cointegrated $C I(d, b)$ if $X_{t}$ has $I(d)$ elements and, for some $b>0$, there exists $\beta$ such that $\beta^{\prime} X_{t}$ is $I(d-b)$. Thus the original definition 
takes $d=b=1$. If either $d$ and/or $b$ is non-integral, we have fractional cointegration. The representation (1) now applies with $F_{t}$ a vector with $I(d)$ components and $V_{t}$ a vector with $I(d-b)$ components.

Some empirical study of fractional cointegration has already been carried out, see e.g. Cheung and Lai (1993), Robinson and Marinucci (1998). However, rigorously justified procedures are currently in short supply, especially in the most practically interesting situation in which $d$ and/or $b$ are unknown. Here, the study of fractional cointegration clearly presupposes a good understanding of statistical inference on $I(d)$ series, in particular on a theory of estimation of $d$. At present this has been much better developed in case of stationary and invertible series, that is when $-1 / 2<d<1 / 2$, than for nonstationary ones, and partly for that reason the present paper focusses principally on the possibility of cointegration in stationary series. This may be of direct interest in financial series, for example of exchange rates between three or more currencies, and some empirical series that have been regarded as having unit roots could be better modelled as $I(d)$ with even $d<1 / 2$. Indeed, Robinson (1994) considered cointegration of stationary $I(d)$ series, showing that $\beta$ can be consistently estimated here, and Robinson and Marinucci (1998) developed his approach. This estimate of $\beta$ converges at only a nonparametric rate, requiring only the assumptions on spectral behaviour of $X_{t}$ entailed in our $I(d)$ definition: defining the spectral density matrix $f(\lambda)$ of $X_{t}$ to satisfy $E\left\{\left(X_{t}-\mu\right)\left(X_{t+j}-\mu\right)^{\prime}\right\}=\int_{-\pi}^{\pi} f(\lambda) e^{i j \lambda} d \lambda$, where $\mu=E\left(X_{t}\right)$, we have

$$
f(\lambda) \backsim G \lambda^{-2 d} \text {, as } \lambda \rightarrow 0+,
$$

where ' $\sim$ ' is taken elementwise, to mean that the ratio of real parts, and of imaginary parts, of left and right sides tends to 1 , and $G$ is a finite nonegative 
definite matrix each of whose diagonal elements is non-null, $G$ being positive definite if and only if $X_{t}$ is not cointegrated (cf Robinson and Marinucci, 1998). Under similar assumptions, with $G$ positive definite, a theory of 'semiparametric' estimation of $d$ has been developed that entails similar convergence rates. More efficient inferences are possible on the basis of parametric models for the autocorrelation in $X_{t}$, such as fractionally integrated autoregressive moving average (FARIMA) models, developed by Adenstedt (1974) and subsequent authors, but model misspecification (under-specifying either the autoregressive or moving average orders or over-specifying both) is liable to lead to inconsistent estimation of fractional differencing and other parameters. Thus the present paper focusses on a semiparametric, low-frequency, approach, as seems natural in the sense that cointegration is esssentially a low-frequency phenomenon, and justifiable insofar as many financial series are sufficiently long that nonparametric rates afford acceptable precision.

A basic question of interest is the existence of cointegration, or, more generally, the value of the cointegrating rank $r$. This problem has been quite well solved in some cases of integer $d, b$, especially $d=b=1$ (see eg Johansen (1996), Phillips and Ouliaris $(1988,1990)$ ). Of course the methods developed there would not necessarily be expected to detect cointegration when in fact alternative values of $d$ and/or $b$ prevail [see e.g. Abadir and Taylor (1999)], and indeed the unit root tests directed against stationary autoregressive (AR) alternatives of Dickey and Fuller (1981) that are commonly employed do not have very good power against fractional alternatives [see e.g., Cheung and Lai (1993), Diebold and Rudebusch (1991)]. While one might envisage some relatively straightforward extension to a fractional context, involving specifying and testing null values of $d$ and $b$ (ideally using 
tests directed against fractional alternatives), it seems preferable to treat $d$ and $b$ as nuisance parameters throughout. Here, while proposals have been made for analyzing cointegration, little rigorous theoretical justification is available. The present paper makes some attempt to redress this situation. We do not discuss estimation of $\beta$ or $b$ in the event of cointegration, and consider estimation of $d$ only for the purposes of testing for cointegration.

The paper is organized as follows. Cointegration requires at least two elements of $X_{t}$ to have the same differencing parameters, but in the following section we discuss a definition of fractional cointegration that allows for some variation in the differencing parameters of elements of $X_{t}$ when $p \geq 3$ (in which case a more general representation than (1) would apply). When all elements have the same differencing parameter, as in (3), $r=p-\operatorname{rank}(G)$. When they do not, on reordering and partitioning the elements of $X_{t}$ into subvectors that have the same differencing parameter, we have a representation of type (3) for the spectral density matrix of each subvector, and the overall cointegrating rank is $p$ minus the sum of the ranks of the "G" matrices for each subvector. Thus the problem essentially reduces to one of determining the rank of $G$ in (3). First, however, in Section 3, we describe a testing algorithm, of a type previously used in the analysis of variance, for the initial partitioning of $X_{t}$; this problem is complicated by the fact that the theory of estimating differencing parameters varies depending on whether or not there is cointegration, which is not known at the partitioning stage. We may then focus on (3) in Section 4, deriving the limit distribution of eigenvalues of an estimate of $G$ under no-cointegration, and thence proposing two methods for determining its rank, one an extension of that of Phillips and Ouliaris (1988) for investigating $\mathrm{CI}(1,1)$ cointegration, the other a model selection proce- 
dure. In Section 5 we apply the procedures to a trivariate series of prices of crude oil. Section 6 contains final comments, including a brief discussion of implications of our work for investigating fractional cointegration in a nonstationary context. Proofs of theoretical results are left to an Appendix.

\section{Fractional integration and cointegration}

We first extend our $I(d)$ definition to $X_{t}$ whose $p$ elements are permitted to have distinct differencing parameters. For $\left|d_{a}\right|<1 / 2, a=1, \ldots, p$, and $\lambda>0$, define the $p \times p$ matrix

$$
\Lambda(\lambda)=\operatorname{diag}\left\{e^{i \pi d_{1} / 2} \lambda^{-d_{1}}, \ldots, e^{i \pi d_{p} / 2} \lambda^{-d_{p}}\right\}
$$

and its complex conjugate $\bar{\Lambda}(\lambda)$.

Definition 1. We call $X_{t}$ an $I\left(d_{1}, \ldots, d_{p}\right)$ series if and only if

$$
f(\lambda) \sim \Lambda(\lambda) G \bar{\Lambda}(\lambda) \text { as } \lambda \rightarrow 0+
$$

and all diagonal elements of the $p \times p$ matrix $G$ are nonzero.

\section{Remark 1}

(i) If $d_{1}=\ldots=d_{p}=d$, then (5) reduces to (3), and so an $I(d, \ldots, d)$ series is a vector of $I(d)$ series.

(ii) We can relate the definition to fractionally differenced time series models such as FARIMAs. Define the $p \times p$ matrix functions

$$
E(L)=\operatorname{diag}\left\{(1-L)^{d_{1}}, \ldots,(1-L)^{d_{p}}\right\}, C(L)=\sum_{j=0}^{\infty} C_{j} L^{j}
$$

for $p \times p$ matrices $C_{j}$ such that $\sum_{j=0}^{\infty} \operatorname{tr}\left\{C_{j} C_{j}^{\prime}\right\}<\infty$, and consider $X_{t}$ given by

$$
E(L)\left(X_{t}-\mu\right)=C(L) e_{t}, \quad t=0, \pm 1, \ldots
$$


where the $p \times 1$ vectors $e_{t}$ are such that $E e_{t}=0, E e_{s} e_{t}^{\prime}=0$ for $s \neq t$, and $E e_{t} e_{t}^{\prime}$ is positive definite, and thus taken with no loss of generality to be the identity matrix. Then

$$
f(\lambda)=E\left(e^{i \lambda}\right)^{-1} C\left(e^{i \lambda}\right) C\left(e^{-i \lambda}\right)^{\prime} E\left(e^{-i \lambda}\right)^{-1} /(2 \pi) .
$$

Now for $\lambda \neq 0, \bmod (\pi)$, and $|d|<1 / 2,\left(1-e^{i \lambda}\right)^{-d}=\sum_{j=0}^{\infty} a_{j}(\cos (j \lambda)+$ $i \sin (j \lambda))$, where $a_{j}=\Gamma(j+d) /(\Gamma(d) \Gamma(j+1))$. Because the $a_{j}$ decrease monotonically to zero and $a_{j} \sim j^{d-1}$ it follows from Theorem III-I of Yong (1974) that as $\lambda \rightarrow 0+,\left(1-e^{i \lambda}\right)^{-d} \rightarrow \lambda^{-d}(\sin ((1-d) \pi / 2)+i \cos ((1-d) \pi / 2))=$ $\lambda^{-d} e^{i d \pi / 2}$. Thus Definition 1 is satisfied with

$$
G=C(1) C(1)^{\prime} /(2 \pi)
$$

if all rows of $C(1)$ are non-null. One choice of $C(L)$ is $C(L)=\Phi(L)^{-1} \Psi(L)$, where $\Phi(L)$ and $\Psi(L)$ are finite-degree polynomial matrices such that all zeros of $\operatorname{det}\{\Phi(z)\}$ and $\operatorname{det}\{\Psi(z)\}$ lie outside the unit circle. Then $X_{t}$ has the vector FARIMA representation

$$
\Phi(L) E(L)\left(X_{t}-\mu\right)=\Psi(L) e_{t}, \quad t=0, \pm 1, \ldots
$$

see eg Lobato (1995), Robinson (1995a). Note that Engle and Granger (1987) defined a vector $I(d)$ series by (10) with $d_{1}=\cdots=d_{p}=d$. With unequal $d_{a}$ we can alternatively define a vector FARIMA as

$$
E(L) \Phi(L)\left(X_{t}-\mu\right)=\Psi(L) e_{t}, \quad t=0, \pm 1, \ldots
$$

(see Lobato (1995)). To relate this to (5) we order elements of $X_{t}$ such that

$$
d_{1}=\cdots=d_{i_{1}}>d_{i_{1}+1}=\cdots=d_{i_{2}}>\cdots>d_{i_{s-1}+1}=\cdots=d_{i_{s}}
$$


where $1 \leq s \leq p, 1 \leq i_{1}<i_{2}<\cdots<i_{s}=p$ with $i_{0}=0$; (12) will also be employed in our discussion of cointegration. Writing $\phi^{a b}$ for the $(a, b)$ th element of $\Phi(1)^{-1}$ and $\psi_{b}^{\prime}$ for the $b$ th row of $\Psi(1)$, define $\theta_{a l}=$ $\sum_{b=i_{l-1}+1}^{i_{l}} \phi^{a b} \psi_{b}$ and $\widetilde{d}_{a}=d_{i_{l(a)}}$, where $l(a)=\arg \max _{l: \theta_{a l} \neq 0} d_{i_{l}}$. Then if $\theta_{a l(a)} \neq 0$, $a=1, \ldots, p$, it follows that $X_{t}$ is an $I\left(\widetilde{d}_{1}, \ldots, \widetilde{d}_{p}\right)$ series, such that in (5) $\Lambda(\lambda)$ is replaced by $\operatorname{diag}\left\{e^{i \pi \widetilde{d}_{1} / 2} \lambda^{-\widetilde{d}_{1}}, \ldots, e^{i \pi \widetilde{d}_{p} / 2} \lambda^{-\widetilde{d}_{p}}\right\}$ and $G=\Theta \Theta^{\prime} / 2 \pi$, where $\Theta=\left(\theta_{1 l(1)}, \ldots, \theta_{p l(p)}\right)^{\prime}$.

If $X_{t}$ is generated by (10) or (11) it cannot be cointegrated under the above conditions on $\Phi(z), \Psi(z)$, but we now discuss cointegration of $I\left(d_{1}, \ldots, d_{p}\right)$ series $X_{t}$. Let $\beta=\left(\beta_{1}, \beta_{2}, \ldots, \beta_{p}\right)^{\prime}$ be a $p$-dimensional vector, and under (12) write

$$
\beta=\left(\beta(1)^{\prime}, \ldots, \beta(s)^{\prime}\right)^{\prime},
$$

where, for $l=1, \ldots, s, \beta(l)=\left(\beta_{i_{l-1}+1}, \ldots, \beta_{i_{l}}\right)^{\prime}$ is a $p_{l}=i_{l}-i_{l-1}$-dimensional vector, conforming to the partition of $d$. We correspondingly write $X_{t}=$ $\left(X_{t}^{(1) \prime}, \ldots, X_{t}^{(s) \prime}\right)^{\prime}$ where $X_{t}^{(l)}=\left(X_{i_{l-1}+1, t}, \ldots, X_{i_{l}, t}\right)^{\prime}$, for $l=1, \ldots, s$.

Definition 2 If there exists a non-null vector $\beta(l)$ such that $\beta(l)^{\prime} X_{t}^{(l)}$ is $I\left(d_{u}\right)$ with $d_{u}<d_{i_{l}}$, then we say $X_{t}$ is cointegrated with cointegrating vector $\beta=\left(0^{\prime}, \ldots, 0^{\prime}, \beta(l)^{\prime}, 0^{\prime}, \ldots 0^{\prime}\right)^{\prime}$. The number of such linearly independent $\beta(l)$ is $r_{l}$, and the cointegrating rank of $X_{t}$ is $r=\sum_{l=1}^{p} r_{l}$.

\section{Remark 2.}

(i) An individual cointegrating vector can be uniquely defined by a normalization. The $r$ cointegrating errors $\beta^{\prime} X_{t}$ can have different differencing parameters $d_{u}$. Our definition does not cover 'polynomial cointegration', in which two or more $X_{t}^{(l)}$ are linked in a cointegrating relation.

(ii) If $d_{1}=\ldots=d_{p}=d$, and $d$ and $d_{u}=d-b$ are integers, our definition is identical with 
$C I(d, b)$ given by Engle and Granger (1987), except that their $I(d)$ definition pertains only to FARIMA series (10).

(iii) The definition implies $p_{l} \geq 2$, but this is not entailed if we adapt the definition of Johansen (1996) (whose treatment concerned only $I(d)$ processes for integer $d$, and in an AR context). Johansen's definition would include as a cointegrating vector any $\beta$ such that $\beta^{\prime} X_{t}$ is $I\left(d_{u}\right)$ for $d_{u}<d_{1}$.

(iv) Flôres and Szafarz (1996) define $X_{t}$ to be cointegrated if there exists $\beta$ with $\beta(1) \neq 0$ such that $\beta^{\prime} X_{t}$ is $I\left(d_{u}\right)$ with $d_{u}<d_{1}$. If Definition 2 provides no cointegrating vector of form $\beta=\left(\beta(1)^{\prime}, 0^{\prime}, \ldots, 0^{\prime}\right)^{\prime}$, then $X_{t}$ is not cointegrated by Flôres and Szafarz's definition.

(v) Robinson and Marinucci (1998) define $X_{t}$ to be cointegrated if there exists $\beta \neq 0$ such that $\beta^{\prime} X_{t}$ is $I\left(d_{u}\right)$ with $d_{u}<d_{p}$.

To illustrate the differing implications of the various definitions, consider the example

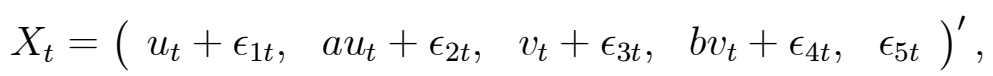

where $a \neq 0, b \neq 0, u_{t}$ is $I(d), v_{t}$ is $I(e)$ with $0<e<d$, and $\epsilon_{i t}, i=1, \ldots, 5$, are $I(0)$. We list the values of $r$ and $\beta$ provided by each of the four definitions:

Definition 2: $r=2 ; \beta=(a,-1,0,0,0)^{\prime},(0,0, b,-1,0)^{\prime}$.

Johansen: $r=4 ; \beta=(a,-1,0,0,0)^{\prime},(0,0, b,-1,0)^{\prime},(0,0,0,1,0)^{\prime},(0,0,0,0,1)^{\prime}$.

Flôres and Szafarz : $r=1 ; \beta=(a,-1,0,0,0)^{\prime}$.

Robinson and Marinucci: $r=0$.

On the other hand if the system consists of only the first two elements of (14) then we get $r=1 ; \beta=(a,-1)^{\prime}$ from all four definitions.

We introduce

Assumption A $X_{t}$ is $I\left(d_{1}, \ldots, d_{p}\right)$. 
Theorem 1. Let Assumption A hold and impose (12). Then if $G_{(l)}$ is the $p_{l} \times p_{l}$ matrix whose $(i, j)$ th element is the $\left(p_{1}+\ldots+p_{l-1}+i, p_{1}+\ldots+p_{l-1}+j\right)$ th element of $G$ (meaning, when $l=1$, the $(i, j)$ th element), then

$$
r_{l}=p_{l}-\operatorname{rank}\left(G_{(l)}\right), \quad r=p-\sum_{l=1}^{s} \operatorname{rank}\left(G_{(l)}\right) .
$$

\section{Remark 3}

Under Definitions 1 and 2 and (12), $r$ can be estimated by a two-stage procedure, to be discussed subsequently, namely first partitioning $X_{t}$ into subvectors $X_{t}^{(l)}, l=1, \ldots, s$, for $1 \leq s \leq p$, such that (12) holds, and then estimating $r_{l}, l=1, \ldots, s$. On the other hand, by aplying a modified version of such a procedure repeatedly, we can also detect polynomial cointegration and cointegration covered by definitions of Flôres and Szafarz (1996), Robinson and Marinucci (1998), but not covered by Definition 2. If Definition 2 has identified cointegrating vectors of form $\left(\beta_{a}(1)^{\prime}, 0^{\prime}, \ldots, 0^{\prime}\right)^{\prime}, 1 \leq a \leq q$, where $1 \leq q<i_{1}$, we may define the $\left(p-i_{1}+q\right) \times 1$ vector $Y_{t}$ to have ath element

$$
Y_{a t}=\left\{\begin{array}{cl}
\beta_{a}(1)^{\prime} X_{t}^{(1)}, & 1 \leq a \leq q, \\
X_{a+i_{1}-q, t}, & q+1 \leq a \leq p-i_{1}+q .
\end{array}\right.
$$

(Of course in practice it is only possible to statistically estimate $q$ and the $\beta_{a}(1)$ from finitely many data.) We might then investigate $Y_{t}$ for cointegration in the same way as we did $X_{t}$, then redefine $Y_{t}$, and continue in this fashion until all cointegrating vectors under these other definitions have been determined. However, we do not pursue this approach, but rather the one referred to at the start of the paragraph. 


\section{Testing the homogeneity of fractional dif- ference parameters}

We propose a specific-to-general type of procedure for partitioning $X_{t}$ into

subvectors $X_{t}^{(l)}, l=1, \ldots, s$, with common differencing parameters, as in (12). A similar procedure is employed in other problems, for example the analysis of variance [see Marcus et al (1976)]. Hsu (1996) provides a comprehensive survey of possible solutions. For $s=1, \ldots, p$, let $\left(\nu_{1}, \nu_{2}, \ldots, \nu_{s}\right)$ be a partition of $(1,2, \ldots, p)$ and define the hypothesis

$H_{\nu_{1} \nu_{2} \ldots \nu_{s}}:\left\{d_{a}=d_{b}\right.$ for $a, b \in \nu_{l}$ and $d_{a} \neq d_{b}$ for $\left.a \in \nu_{l}, b \in \nu_{l^{\prime}}, l \neq l^{\prime}, l, l^{\prime}=1, \ldots, s\right\}$.

We first take $s=1$ and test

$$
H_{\nu_{1}}: d_{1}=d_{2}=\ldots=d_{p}
$$

If $H_{\nu_{1}}$ is not rejected, the procedure terminates. If $H_{\nu_{1}}$ is rejected, we test each hypothesis $H_{\nu_{1} \nu_{2}}$ and terminate if at least one of these is not rejected. Otherwise, we proceed to test each hypothesis $H_{\nu_{1} \nu_{2} \nu_{3}}$, and so on. If we eventually reach $s=p-1$, and then reject each $H_{\nu_{1} \nu_{2} \ldots \nu_{p-1}}$, we can conclude there is no cointegration, and so in no case do we test $H_{\nu_{1} \nu_{2} \ldots \nu_{p}}$. Thus, we test at most $\sum_{s=1}^{p} S(p, s)-1$ hypotheses, where $S(p, s)=\frac{1}{s !} \sum_{i=0}^{s}(-1)^{i}\left(\begin{array}{l}s \\ i\end{array}\right)(s-i)^{p}$ is the Stirling number of the second kind [see Liu (1968), pp.38-39]. On the other hand if we do not reject more than one of $H_{\nu_{1} \nu_{2} \ldots \nu_{s}}$, for some $s=2, \ldots, p-1$, then we can either investigate each of the corresponding subvectors of $X_{t}$, or else select the one with the most insignificant test statistic.

We now consider the testing of $H_{\nu_{1} \nu_{2} \ldots \nu_{s}}$. Noting that $H_{\nu_{1} \nu_{2} \ldots \nu_{s}}$ is a joint hypothesis, composed of the hypotheses $H_{\rho_{l}}, l=1, \ldots, s$, where

$$
H_{\rho_{l}}:\left\{d_{a} \text { are equal for } a \in \nu_{l}\right\}
$$


we test each such $H_{\rho_{l}}$ individually and reject $H_{\nu_{1} \nu_{2} \ldots \nu_{l}}$ if and only if at least one is rejected. Of course, since we do not need to test $H_{\rho_{l}}$ with $n_{l}=\#\left\{\nu_{l}\right\}=1$, actually $H_{\nu_{1} \nu_{2} \ldots \nu_{s}}$ is composed only of $H_{\rho_{l}}$ such that $n_{l}>1$.

A test of $H_{\rho_{l}}$ can be based on $\hat{d}_{a}-\hat{d}_{b}$, for $a<b, a, b \in \nu_{l}$, where $\hat{d}_{a}, \hat{d}_{b}$ are estimates of $d_{a}, d_{b}$. Several consistent semiparametric estimates of differencing parameters have been proposed [see e.g.Geweke and Porter-Hudak (1983), Künsch (1987), Robinson (1994,1995a,b)]. We shall use the Gaussian semiparametric or local Whittle estimate of Künsch(1987), Robinson(1995b), because it has similarly nice asymptotic properties to the log periodogram estimate, but is more efficient..

Given observations $X_{t}, t=1,2, \ldots, n$, introduce the discrete Fourier transform

$$
w_{a}(\lambda)=\frac{1}{\sqrt{2 \pi n}} \sum_{t=1}^{n} X_{a t} e^{i t \lambda}, a=1, \ldots, p,
$$

where mean-correction is unnecessary because $w_{a}(\lambda)$ will be computed only at frequencies $\lambda_{j}=2 \pi j / n$ for $j=1, \ldots, m<n / 2$. The (cross-)periodogram of $X_{a t}$ and $X_{b t}$ is

$$
I_{a b}(\lambda)=w_{a}(\lambda) w_{b}(-\lambda) .
$$

For brevity we write $I_{a b, j}$ for $I_{a b}\left(\lambda_{j}\right)$. Define

$$
\hat{d}_{a}=\arg \min _{\Delta \in\left[\Delta_{1}, \Delta_{2}\right]} R_{a}(\Delta),
$$

where $\Delta_{1}$ and $\Delta_{2}$ satisfy $-1 / 2<\Delta_{1}<\Delta_{2}<1 / 2$, and

$$
R_{a}(\Delta)=\log \left(\frac{1}{m} \sum_{j=1}^{m} j^{2 \Delta} I_{a a, j}\right)-2 \Delta \frac{1}{m} \sum_{j=1}^{m} \log j .
$$

We introduce the following further assumptions.

Assumption B. Defining $A(L)=\sum_{j=0}^{\infty} A_{j} L^{j}$, where the $A_{j}$ are $p \times p$ matrices,

$$
X_{t}=\mu+A(L) e_{t}, \quad t=0, \pm 1, \ldots
$$


where, almost surely, $E\left(e_{t} \mid \mathcal{F}_{t-1}\right)=0, E\left(e e_{t}^{\prime} \mid \mathcal{F}_{t-1}\right)=I_{p}$, the $p \times p$ identity matrix, and the matrices $E\left(e_{t} \otimes e_{t} e_{t}^{\prime} \mid \mathcal{F}_{t-1}\right), E\left(e_{t} e_{t} \otimes e_{t} e_{t}^{\prime} \mid \mathcal{F}_{t-1}\right)$ are finite, nonstochastic and constant in $t, \mathcal{F}_{t}$ being the $\sigma$-field of events generated by $e_{s}, s \leq t$

Assumption C. Writing $A_{a b}(L)$ for the $(a, b)$ th element of $A(L)$,

$$
\frac{d}{d \lambda} A_{a b}\left(e^{i \lambda}\right)=O\left(\left\{\left|A_{a a}\left(e^{i \lambda}\right)\right|\left|A_{b b}\left(e^{i \lambda}\right)\right|\right\}^{1 / 2} / \lambda\right) \text { as } \lambda \rightarrow 0+,
$$

for $a, b=1, \ldots, p$.

Assumption D. With $C(L)=E(L) A(L)$ as in (6), (7), we have for $\xi \in(0,2]$,

$$
C\left(e^{i \lambda}\right)=C(1)\left(1+O\left(\lambda^{\xi}\right)\right) \text { as } \lambda \rightarrow 0+
$$

Assumption E. For the same $\xi$ as in Assumption D,

$$
\frac{1}{m}+\frac{m^{1+2 \xi}(\log m)^{2}}{n^{2 \xi}} \rightarrow 0 \text { as } n \rightarrow \infty
$$

\section{Assumption F.}

$$
d_{a} \in\left(\Delta_{1}, \Delta_{2}\right), a=1, \ldots, p
$$

Assumptions B, C and E are the same as assumptions A2, A3 and A4 of Lobato (1999)respectively, and are analogous to assumptions A3', A2' and A4' of Robinson (1995b) for scalar $X_{t}$. Assumption D implies that the error in approximating the left side of (3) by the right is $O\left(\lambda^{\xi}\right)$, which is a similar assumption to A1' of Robinson (1995b) and A1 of Lobato (1999). The case $\xi=2$ aplies to FARIMA models, for example, and is standard in other circumstaces of smoothed nonparametric estimation, such as probability and spectral density estimation. The interior-point Assumption $\mathrm{F}$ is standard in central limit theory for 
implicitly-defined extremum estimates.

For the sequel we redefine $d$ as the $p \times 1$ vector $d=\left(d_{1}, \ldots, d_{p}\right)^{\prime}$. Define also $\widehat{d}=\left(\widehat{d}_{1}, \ldots, \widehat{d}_{p}\right)^{\prime}$ and $D=\operatorname{diag}\left\{G_{11}, \ldots, G_{p p}\right\}$ where $G_{a b}$ is the $(a, b)$ th element of $G$, and denote Hadamard product by $\circ$ and transposition combined with complex conjugation by ${ }^{*}$.

Proposition 1 Under Assumptions A-F,

$$
m^{1 / 2}(\hat{d}-d) \rightarrow_{d} N\left(0, \frac{1}{4} D^{-1}(G \circ G) D^{-1}\right) \text { as } n \rightarrow \infty .
$$

$A$ consistent estimate of $G$ is

$$
\widehat{G}=\frac{1}{m} \sum_{j=1}^{m} \operatorname{Re}\left\{\widehat{\Lambda}\left(\lambda_{j}\right)^{-1} I_{j} \widehat{\Lambda}\left(\lambda_{j}\right)^{-1 *}\right\},
$$

where $I_{j}=I\left(\lambda_{j}\right), I(\lambda)=w(\lambda) w(\lambda)^{*}, w(\lambda)=\left(w_{1}(\lambda), \ldots, w_{p}(\lambda)\right)^{\prime}$, and $\widehat{\Lambda}(\lambda)$ is $\Lambda(\lambda)$ with $d$ replaced by $\widehat{d}$.

We apply this result to a single test of pairwise equality

$$
H_{a b}: d_{a}=d_{b}
$$

Denote by $G_{a b}, \widehat{G}_{a b}$ the $(a, b)$ th elements of $G, \widehat{G}$ respectively. Noting that $G_{a a} G_{b b}-G_{a b}^{2}$ is the determinant of the matrix formed by omitting from $G$ all rows and columns but the $a$ th and $b$ th, if $X_{a t}$ and $X_{b t}$ are cointegrated $G_{a b}^{2}=G_{a a} G_{b b}$, while if they are not, $G_{a b}^{2}<G_{a a} G_{b b}$. It thus follows from Proposition 1 and the delta method that under $H_{a b}$ the limit distribution of

$$
\widetilde{T}_{a b}=m^{1 / 2}\left(\hat{d}_{a}-\hat{d}_{b}\right) /\left\{\frac{1}{2}\left(1-\widehat{G}_{a b}^{2} /\left(\widehat{G}_{a a} \widehat{G}_{b b}\right)\right)\right\}^{1 / 2}
$$

is standard normal if $X_{a t}$ and $X_{b t}$ are not cointegrated, but is not well defined if they are. Since the presence or absence of cointegration is not known at the time of testing $H_{a b}$, we require a test statistic that is informative under 
both circumstances. Define

$$
\hat{T}_{a b}=\frac{m^{1 / 2}\left(\hat{d}_{a}-\hat{d}_{b}\right)}{\left\{\frac{1}{2}\left(1-\widehat{G}_{a b}^{2} /\left(\widehat{G}_{a a} \widehat{G}_{b b}\right)\right)\right\}^{1 / 2}+h(n)},
$$

where $h(n)>0$. We introduce

\section{Assumption G}

$$
h(n)+\frac{(\log m) m^{1 / 2+\xi} / n^{\xi}+(\log m)^{2} m^{-1 / 6}}{h(n)} \rightarrow 0, \text { as } n \rightarrow \infty .
$$

Theorem 2 Let Assumptions $A-G$ hold. Then under $H_{a b}(19)$, as $n \rightarrow$ $\infty$ :

(i) If $X_{a t}$ and $X_{b t}$ are not cointegrated, $\hat{T}_{a b} \rightarrow{ }_{d} N(0,1)$;

(ii) If $X_{a t}$ and $X_{b t}$ are cointegrated, $\hat{T}_{a b} \rightarrow_{p} 0$.

Of course $\hat{T}_{a b}$ is potentially highly sensitive to choice of $h(n)$, but a significantly large value of $\left|\hat{T}_{a b}\right|$, relative to the $N(0,1)$ distribution, can be taken as evidence against $H_{a b}$, irrespective of whether or not there is cointegration, whereas a decision of non-rejection under $h(n)=0$ (i.e. based on $\widetilde{T}_{a b}$ ) would be made with greater confidence for any positive $h(n)$.

To test $H_{\rho_{l}}$ we can consider

$$
\hat{T}_{\rho_{l}}=\max _{a, b \in \nu_{l}}\left|\widehat{T}_{a b}\right|
$$

The level of the test of each $H_{\rho_{l}}$ must provide a level- $\alpha$ test of $H_{\nu_{1} \nu_{2} \ldots \nu_{s}}$. Unlike in classical analysis of variance, the test statistics $\hat{T}_{\rho_{l}}$ are not mutually independent. For $s^{\prime}=\#\left\{l: n_{l}>1, l=1, \ldots, s\right\}$, a level- $\left(1-(1-\alpha)^{1 / s^{\prime}}\right)$ test based on $\hat{T}_{\rho_{l}}$ for each $H_{\rho_{l}}$ does thus not necessarily assure a level- $\alpha$ test for $H_{\nu_{1} \nu_{2} \ldots \nu_{s}}$. However by the Bonferroni inequality, if a level- $\alpha / s^{\prime}$ test for $H_{\rho_{l}}$ with $n_{l}>1$ is given by $\hat{T}_{\rho_{l}}$, which in turn is given by a level-2 $\alpha /\left(s^{\prime} n_{l}\left(n_{l}-1\right)\right)$ test for $H_{a b}$ based on the $n_{l}\left(n_{l}-1\right) / 2$ statistics $\left|\hat{T}_{a b}\right|, a<b, a, b \in \nu_{l}$, it assures a level- $\alpha$ test for $H_{\nu_{1} \nu_{2} \ldots \nu_{s}}$. Specifically, if we reject $H_{\nu_{1} \nu_{2} \ldots \nu_{s}}$ when at least 
one of $\left|\hat{T}_{a b}\right|, a<b, a, b \in \nu_{l}, l=1, \ldots, s$, exceeds $z_{\alpha /\left(s^{\prime}\left(n_{l}\left(n_{l}-1\right)\right)\right.}$ where $z_{c}$ is the $1-c$ quantile of $N(0,1)$, we achieve, asymptotically, a level- $\alpha$ test for $H_{\nu_{1} \nu_{2} \ldots \nu_{s}}$. An alternative approach, which avoids a user-chosen sequence such as $h(n)$, involves applying in the same way

$$
\bar{T}_{a b}=(2 m)^{1 / 2}\left(\hat{d}_{a}-\hat{d}_{b}\right), \quad \bar{T}_{\rho_{l}}=\max _{a, b \in \nu_{l}}\left|\bar{T}_{a b}\right|
$$

which is more conservative than $\hat{T}_{\rho_{l}}$ because the asymptotic variance of $\bar{T}_{a b}$ is generally less than one.

According to the above pairwise approach, $n_{l}\left(n_{l}-1\right) / 2$ separate tests are involved in the testing of $H_{\rho_{l}}$ alone. These ideas can be extended, when $n_{l} \geq 3$, to permit a single test of $H_{\rho_{l}}$. We can write $H_{\rho_{l}}$ as $S d=0$, where, with $\nu_{l}=\left(a_{1}, \ldots, a_{n_{l}}\right), S$ is the $\left(n_{l}-1\right) \times p$ matrix whose $j$ th row has 1 as its $a_{j}$ th element and -1 as its $a_{j+1}$ th element and the remainder 0 , $j=1, \ldots, n_{l}-1$. Denote $\widehat{D}=\operatorname{diag}\left\{\widehat{G}_{11}, \ldots, \widehat{G}_{p p}\right\}$. Then, for example, in view of Proposition 1, under $H_{\rho_{l}}$ the statistic

$$
(S \widehat{d})^{\prime}\left(S \frac{1}{4} \widehat{D}^{-1}(\widehat{G} \circ \widehat{G}) \widehat{D}^{-1} S^{\prime}+h(n)^{2} I_{n_{l}-1}\right)^{-1}(S \widehat{d})
$$

has a limiting $\chi_{n_{l}-1}^{2}$ distribution when there is no cointegration, and converges in probability to zero otherwise.

\section{Determination of fractional cointegrating rank}

We now suppose that $X_{t}$ has already been partitioned into subvectors $X_{t}^{(l)}, l=$ $1, \ldots, s$, satisfying (12), perhaps by applying the procedures of the previous section. We shall only attempt to estimate the cointegrating ranks of such $X_{t}^{(l)}$ individually, in which case for notational convenience we can take $s=1$, 
so that (16) holds, and consider the cointegrating rank of $X_{t}$ itself. We denote by $d_{*}$ the common value of $d_{1}, \ldots, d_{p}$. In view of $(3)$ and Theorem 1 we commence by obtaining estimates of $G$ and its eigenvalues, and determine their limit distribution. Consider (cf (18))

$$
\hat{G}\left(d_{*}\right)=\frac{1}{m} \sum_{j=1}^{m} \lambda_{j}^{2 d_{*}} \operatorname{Re}\left(I_{j}\right) .
$$

Note that $\pi \hat{G}(0)$ was used by Phillips and Ouliaris (1988) in testing the cointegrating rank of an $I(1)$ vector. Let $G_{a}$ be the ath column of $G$.

Proposition 2. Let Assumptions A-G hold. Then as $n \rightarrow \infty$

$$
m^{1 / 2} \operatorname{vec}\left(\hat{G}\left(d_{*}\right)-G\right) \rightarrow_{d} N\left(0, \frac{1}{2}\left(G \otimes G+\left(G \otimes G_{1}, \ldots, G \otimes G_{p}\right)\right)\right) .
$$

Since $d_{*}$ is unknown this result is not of direct use. We might think of estimating $d_{*}$ by versions of the multivariate log periodogram method of Robinson (1995a) or multivariate local Whittle method (cf (17)) of Lobato (1995, 1999) that gain efficiency by exploiting the fact that the elements of $X_{t}$ have the same differencing parameters; denoting such an estimate $\widehat{d}_{*}$, we might then estimate $G$ by $\hat{G}\left(\widehat{d}_{*}\right)$. There are two problems with this approach. First, if $\widehat{d}_{*}$ uses the same bandwidth $m$ as in (20) then, as found by Robinson (1995a), $\hat{G}\left(\widehat{d}_{*}\right)$ does not have the limit distribution of $(21)$, but rather, though it is normal, it is undesirably asymptotically perfectly correlated with $\widehat{d}_{*}$ and indeed is only $m^{1 / 2} / \log n$-consistent. Second, these results of Robinson (1995a), Lobato (1995, 1999) assume $G$ has full rank and are thus not valid if $X_{t}$ is cointegrated. We deal with both difficulties by instead pooling estimates of $d_{*}$ based on the individual elements of $X_{t}$ (which make no presumption about cointegration) and computing the latter 
with a bandwidth $m_{1}$ that increases sufficiently faster than $m$ that the effect of estimating $d_{*}$ has no effect on $(21)$. Denote by $\widetilde{d}_{a}$ the estimate $d_{a}$ given by (17) but with $m$ replaced by $m_{1}$ and define

$$
\bar{d}_{*}=\frac{1}{p} \sum_{a=1}^{p} \widetilde{d}_{a}
$$

Assumption $\mathbf{H}$ For any $\zeta>0$

$$
\frac{m^{1 / 2-\zeta} n^{\zeta}}{m_{1}^{1 / 2}}+\frac{m_{1}^{1+2 \xi}\left(\log m_{1}\right)^{2}}{n^{2 \xi}} \rightarrow 0 \text {, as } n \rightarrow \infty .
$$

Proposition 3. Let Assumptions $A-F$ and $H$ hold. Then as $n \rightarrow \infty$,

$$
m^{1 / 2} \operatorname{vec}\left(\hat{G}\left(\bar{d}_{*}\right)-G\right) \rightarrow_{d} N\left(0, \frac{1}{2}\left(G \otimes G+\left(G \otimes G_{1}, \ldots, G \otimes G_{p}\right)\right)\right)
$$

Assumption I. $\operatorname{rank}(G)=p-r$, for $0 \leq r<p$, and the nonzero eigenvalues of $G$ are distinct.

Let $\delta_{a}\left(\widehat{\delta}_{a}\right)$ be the ath eigenvalue of $G\left(\hat{G}\left(\bar{d}_{*}\right)\right), a=1, \ldots, p$, ordered such that $\delta_{1}>\delta_{2}>\cdots>\delta_{p-r}>0$, with $\delta_{p-r+1}=\ldots=\delta_{p}=0$, for $r \geq 1$, and $\widehat{\delta}_{1} \geq \widehat{\delta}_{2}>\cdots \geq \widehat{\delta}_{p}$

Proposition 4 Let Assumptions A-F, $H$ and I hold. Then the $m^{1 / 2}\left(\widehat{\delta}_{a}-\right.$ $\left.\delta_{a}\right)$ are asymptotically independent for $a=1, \ldots, p$, converge in distribution to $N\left(0, \delta_{a}^{2}\right)$ variates for $a=1, \ldots, p-r$, and are $o_{p}(1)$ for $a=p-r+1, \ldots, p$.

Proposition 4 can be interpreted as a variant of Theorem 9.4.4 of Brillinger (1975) as $m$ and $n$ tend to infinity simultaneously, and suggests that Theorem 1 of Anderson (1963) and Theorem 13.5.1 of Anderson (1984) are still true in the singular case..

Now define, for $j=1, \ldots, p-1$,

$$
\pi_{j}=\frac{\sigma_{p-j+1, p}^{(1)}}{\sigma_{1, p}^{(1)}}, \quad \widehat{\pi}_{j}=\frac{\widehat{\sigma}_{p-j+1, p}^{(1)}}{\widehat{\sigma}_{1, p}^{(1)}}
$$


where

$$
\sigma_{k, l}^{(i)}=\sum_{a=k}^{l} \delta_{a}^{i}, \quad \widehat{\sigma}_{k, l}^{(i)}=\sum_{a=k}^{l} \widehat{\delta}_{a}^{i}
$$

Also define

$$
s_{j}=\frac{\left(\widehat{\sigma}_{p-j+1, p}^{(1) 2} \widehat{\sigma}_{1, p-j}^{(2)}+\widehat{\sigma}_{1, p-j}^{(1) 2} \widehat{\sigma}_{p-j+1, p}^{(2) 2}\right)^{1 / 2}}{\widehat{\sigma}_{1, p}^{(1) 2}} .
$$

Theorem 3 Let Assumptions $A-F, H$ and $I$ hold, and let $r=0$. Then for $j=1, \ldots, p-1$

$$
m^{1 / 2}\left(\widehat{\pi}_{j}-\pi_{j}\right) / s_{j} \rightarrow{ }_{d} N(0,1) \text {, as } n \rightarrow \infty
$$

The application of Theorem 3 in determining $r$ by hypothesis testing is hampered by the assumption that $r=0$. We propose two rather ad hoc solutions, both of which might be applied for increasing values of $r$ :

(i) This directly follows the proposal of Phillips and Ouliaris (1988) for the case of $C I(1,1)$ cointegration. To test that the cointegrating rank is $r$ we consider the $100(1-\alpha) \%$ upper confidence interval for $\pi_{r}$ based on Theorem 3, namely

$$
\widehat{\pi}_{r}+s_{r} z_{\alpha} / m^{1 / 2}
$$

We find evidence in favour of the hypothesis that the cointegrating rank is $r$ if this is smaller than some prescribed threshold, such as $0.1 / p$ (as sugggested by Phillips and Ouliaris (1988)).

(ii). We can apply Theorem 3 to test that $\pi_{r}$ is some sufficiently small positive value $\gamma$, eg $\gamma=0.01 / p$. Then if we reject this hypothesis in favour of the alternative $\pi_{r}>\gamma$ we find evidence against a cointegrating rank of $r$. The specification of a small null value of $\pi_{r}$ might seem more attractive than the specification of a threshold for (22), but one expects that the central limit theory of Theorem 3 for $\widehat{\delta}_{a}$ may provide a poor approximation as $\delta_{a} \rightarrow 0$. 
Alternatively, we may consider a model selection procedure which consistently estimates $r$ [cf Fujikoshi (1985), Fujikoshi and Veitch (1979), Gunderson and Muirhead (1997)]. Define, for $v(n)>0$,

$$
L(u)=v(n)(p-u)-\widehat{\sigma}_{1, p-u}^{(1)},
$$

and estimate $r$ by

$$
\widehat{r}=\arg \min _{u=1, \ldots, p-1} L(u) .
$$

\section{Assumption J}

$$
v(n)+\frac{1}{m^{1 / 2} v(n)} \rightarrow 0, \text { as } n \rightarrow \infty
$$

Theorem 4. Let Assumptions A-F and H-J hold. Then

$$
\lim _{n \rightarrow \infty} P(\widehat{r}=r)=1
$$

If elements of $X_{t}$ are measured in different units, scale-invariant statistics might seem preferable, as sugggested in the $C I(1,1)$ case by Phillips and Ouliaris (1988). For example, we might wish to base the analysis not on $\hat{G}\left(\bar{d}_{*}\right)$ but rather on the correlation matrix $\hat{P}\left(\bar{d}_{*}\right)=\widehat{D}\left(\bar{d}_{*}\right)^{-1 / 2} \hat{G}\left(\bar{d}_{*}\right) \widehat{D}\left(\bar{d}_{*}\right)^{-1 / 2}$, where $\widehat{D}\left(\bar{d}_{*}\right)$ is the diagonal matrix whose ath diagonal element is the same as that of $\hat{G}\left(\bar{d}_{*}\right)$. Unfortunately the limiting covariance structure of the eigenvalues of $\hat{P}\left(\bar{d}_{*}\right)$ is much more complicated than of those of $\hat{G}\left(\bar{d}_{*}\right)$, and so testing procedures of corresponding simplicity to those derived from Theorem 3 are not available. However, because its probability limit has the same rank as $\hat{G}\left(\bar{d}_{*}\right), \hat{P}\left(\bar{d}_{*}\right)$ can be used in a model choice procedure analogous to that justified in Theorem 4. 


\section{Empirical example}

We apply the procedure developed in the preceding sections to a trivariate series of 146 observations on spot closing prices of crude oil, namely West Texas Intermediate(WTI), Brent, and Dubai (which are said to be key markers in the US, European and Asian markets respectively) recorded on the last trading day of each month from January 1986 through February 1998. (The price of WTI for October 1991 was not observed due to a pipeline accident and is replaced by the mean of the September and November 1991 observations). We analyze $\log$ prices, taking $p=3$ and $X_{1 t}=\log \mathrm{WTI}_{t}, X_{2 t}=\log$ Brent $_{t}$ and $X_{3 t}=\log$ Dubai $_{t}$ in the notation of the paper.

Since unit root analysis in an AR setting is standard in econometrics, we commenced in this fashion, using the model

$$
X_{a t}=c+\rho_{a} X_{a, t-1}+\sum_{i=1}^{N_{a}-1} \phi_{a i}\left(X_{a, t-i}-X_{a, t-1-i}\right)+\epsilon_{a t}, a=1,2,3 .
$$

We applied the augmented Dickey-Fuller test [see e.g. Dickey and Fuller (1981), Said and Dickey(1984)] because the series (see Figure 1) appear to have nonzero mean but reveal no distinctive seasonal pattern or trend except for the upturn around the period of the Gulf War from late 1990 to early 1991. Choosing the $\mathrm{AR}$ order $N_{a}$ to attain the minimum AIC between 0 and 15, we obtained $N_{1}=11, N_{2}=9$ and $N_{3}=5$. Table 1 shows $t$-ratios for $\rho_{a}-1$ with $5 \%$ and $1 \%$ critical values, based on Table 1 of MacKinnon (1991), allowing for a constant but no trend. We rejected the unit root null hypothesis at $1 \%$ for WTI and Dubai and at 5\% for Brent.

The AR orders chosen by AIC are on the large side, particularly for WTI and Brent, and so stationary long memory is an alternative possibility. Table 2 presents the estimates $\widehat{d}_{a}, a=1,2,3$ of (17), using $m=20$ and 
$m=15$,along with standard errors, obtained from the asymptotic variance formula $1 / 4 m$ for univariate estimates (cf Proposition 1). With the exception of WTI with $m=20$, all estimates are less than 0.5 . We focus on the estimates with $m=15$, which are consistent with stationarity.

In Table 3 we report $\widehat{G}$ (18) with $m=13$, thereby deducing the test statistics $\widetilde{T}_{12}=2.626, \widetilde{T}_{13}=2.768$ and $\widetilde{T}_{23}=1.273$. Thus $H_{123}: d_{1}=d_{2}=d_{3}$ is not rejected at $1 \%$, and would be more strongly supported by $\widehat{T}_{12}, \widehat{T}_{13}$ and $\widehat{T}_{23}$ with $h(m)>0$, while $H_{23}: d_{2}=d_{3}$ is not rejected at level $5 \%$.

In consequence we investigate the presence of cointegration on the basis of both $s=1$ and $s=2$, where in the latter case Brent and Dubai are supposed to have a common differencing parameter. Table 4 considers the case $s=1$, showing $\hat{G}\left(\bar{d}_{*}\right)$ and its eigenvalues and those of $\hat{P}\left(\bar{d}_{*}\right)$, while Table 5 considers $s=2$, showing the $2 \times 2$ matrix $\hat{G}_{(1)}\left(\bar{d}_{*}\right)$ and its eigenvalues and those of $\hat{P}_{(1)}\left(\bar{d}_{*}\right), \hat{G}_{(1)}\left(\bar{d}_{*}\right)$ estimating $G_{(1)}$ and $\hat{P}_{(1)}\left(\bar{d}_{*}\right)$ thence defined analogously to $\hat{P}\left(\bar{d}_{*}\right)$; in each case $m=13, m_{1}=15$. The largest eigenvalue greatly dominates throughout, so that for any reasonable value of $\delta$ the objective function $L(u)$ will support the conclusion $r=2$ when $s=1$, and $r=1$ when $s=2$. Also, with $r=2$ and $\alpha=0.05,(22)$ is $0.0338 \backsim 1 / 30=0.10 / p$. This is the threshold suggested by Phillips and Ouliaris (1988) so that the conclusion concerning $r=2$ is rather uncertain. Since $H_{23}$ seemed more strongly supported than $H_{123}$ we might thus prefer $r=1$, for which Table 5 provides support.

\section{Final Comments}

1. The procedures suggested all involve choice of a bandwidth $m$ (and also $m_{1}$ involved in $\bar{d}_{*}$ ). User-chosen bandwidth numbers are inevitable in smoothed 
nonparametric estimation, and as usual the results will be sensitive to the choice made. Some proposals for choosing $m$ in (17) are made by Henry and Robinson (1996).

2. Perhaps more seriously, the procedures also depend on other userchosen numbers, namely $h(n), v(n)$ and the threshold for (22) (or the null hypothesis on $\pi_{r}$ ). Our introduction of these is an indication of the difficulty of the problems tackled, but clearly it would be desirable to develop more objective methods.

3. There is interest in extending the methods to cover nonstationary $X_{t}$. The extension of Definition 2 here is straightforward. If, on the other hand, we have sufficient prior knowledge to first-difference the raw series to the stsationary/invertible region $(-1 / 2,1 / 2)$, then our results may be applicable, though this may depend also on the differenced cointegrating errors having differencing parameters that lie in this region. If we then go on to estimate $\beta$ it is important that the undifferenced series be used here in order to achieve a fast rate of convergence [see Robinson and Marinucci (1998)] So far as applicability of our present procedures to raw nonstationary $X_{t}$ is concerned, Velasco (1999) showed that in case of scalar series, at least suitably tapered versions of the estimates (17) can still be $m^{1 / 2}$ - consistent and asymptotically normal. Thus it seems that suitable modifications of our procedures may directly apply to nonstationary $X_{t}$, though it remains to provide rigorous justification.

\section{Appendix:Proofs}

Proof of Theorem 1. From (3) the spectral density matrix $f_{\beta_{l}}(\lambda)$ of 
$\beta^{\prime}(l) X_{t}^{(l)}$ satisfies

$$
f_{\beta_{l}}(\lambda) \backsim \lambda^{-2 d_{l}} \beta^{\prime}(l) G_{(l)} \beta(l), \text { as } \lambda \rightarrow 0+.
$$

Hence $\beta(l)$ is a cointegrating vector for $X_{t}^{(l)}$ if and only if $\beta^{\prime}(l) G_{(l)} \beta(l)=0$. Then (15) follows immediately.

Proof of Proposition 1. We only briefly summarize the proof since it follows like that of Theorem 2 of Robinson (1995b). We write $R_{a}^{(i)}(\Delta)=$ $\partial^{i} R_{a}(\Delta) / \partial \Delta^{i}$. With probability approaching 1 , as $n \rightarrow \infty, \hat{d}_{a}$ satisfies

$$
0=R_{a}^{(1)}\left(\widehat{d}_{a}\right)=R_{a}^{(1)}\left(d_{a}\right)+R_{a}^{(2)}\left(\bar{d}_{a}^{*}\right)\left(\hat{d}_{a}-d_{a}\right)
$$

where $\left|\bar{d}_{a}^{*}-d_{a}\right| \leq\left|\hat{d}_{a}-d_{a}\right|$. Now

$$
m^{1 / 2}\left(R_{1}^{(1)}\left(d_{1}\right), \ldots, R_{p}^{(1)}\left(d_{p}\right)\right)^{\prime} \rightarrow_{d} N\left(0,4 D^{-1 / 2}(G \circ G) D^{-1 / 2}\right)
$$

and $R_{a}^{(2)}\left(\bar{d}_{a}^{*}\right) \rightarrow_{p} 4$, whence the result follows immediately.

Proof of Theorem 2. The proof of (i) follows quickly from Proposition 1 and $h(n) \rightarrow 0$. To prove (ii) it suffices, from Assumption G, to show that $\hat{d}_{a}-\hat{d}_{b}=O_{p}\left((\log m)(m / n)^{\xi}+(\log m)^{2} m^{-2 / 3}\right)$. Note first that under $H_{a b}$, with $d_{a}=d_{b}=d_{*}$, the mean value theorem gives, with probability approaching 1 as $n \rightarrow \infty$,

$\hat{d}_{a}-\hat{d}_{b}=\left(\frac{R_{b}^{(1)}\left(d_{*}\right)}{R_{b}^{(2)}\left(d_{*}\right)}-\frac{R_{a}^{(1)}\left(d_{*}\right)}{R_{a}^{(2)}\left(d_{*}\right)}\right)-\frac{1}{2}\left(\frac{R_{a}^{(3)}\left(\bar{d}_{a}\right)}{R_{a}^{(2)}\left(d_{*}\right)}\left(\hat{d}_{a}-d_{*}\right)^{2}-\frac{R_{b}^{(3)}\left(\bar{d}_{b}\right)}{R_{b}^{(2)}\left(d_{*}\right)}\left(\hat{d}_{b}-d_{*}\right)^{2}\right)$,

where $\left|\bar{d}_{a}-d_{*}\right| \leq\left|\widehat{d}_{a}-d_{*}\right|,\left|\bar{d}_{b}-d_{*}\right| \leq\left|\widehat{d}_{b}-d_{*}\right|$. The second term on the right is $O_{p}\left(m^{-1}\right)$, by the same argument as that of Theorem 2 of Robinson (1995b) and, for all $a, R_{a}^{(2)}\left(d_{*}\right) \rightarrow_{p} 4, R_{a}^{(3)}\left(\bar{d}_{*}\right) \rightarrow_{p}-16$ and $\hat{d}_{a}-d_{*}=O_{p}\left(m^{-1 / 2}\right)$. The first term is

$$
\frac{R_{b}^{(1)}\left(d_{*}\right)-R_{a}^{(1)}\left(d_{*}\right)}{R_{a}^{(2)}\left(d_{*}\right)}+R_{b}^{(1)}\left(d_{*}\right)\left(\frac{1}{R_{b}^{(2)}\left(d_{*}\right)}-\frac{1}{R_{a}^{(2)}\left(d_{*}\right)}\right) .
$$


As found by Robinson (1995b), $R_{a}^{(i)}\left(d_{*}\right)$ is scale-free, so bearing in mind also that $G_{a b}^{2}=G_{a a} G_{b b}$, we may take

$$
G_{a a}=\left|G_{a b}\right|=G_{b b}=1
$$

Also as found by Robinson $(1995 \mathrm{~b}), R_{a}^{(i)}\left(d_{*}\right)$ is a differentiable function of

$$
Y_{a}^{(l)}=\frac{1}{m} \sum_{j=1}^{m}(\log j)^{l} I_{a a, j} \lambda_{j}^{2 d}, l=0,1, \ldots, i .
$$

As shown by Robinson (1995b) and Lobato (1999,Appendix C), for $l \geq 0$, $Y_{a}^{(l)}$ differs from

$$
Z_{a}^{(l)}=\frac{1}{m} \sum_{j=1}^{m}(\log j)^{l} C_{a}^{\prime} J_{j} C_{a}
$$

by $O\left((\log m)^{l}(m / n)^{\xi}+(\log m)^{l+1} m^{-2 / 3}\right), l=0,1, \ldots, i$, where $C_{a}^{\prime}$ is the ath row of $C(1)$ and $J_{j}$ is defined like $I_{j}$ but with $e_{t}$ replacing $X_{t}$. Thus consideration of $R_{a}^{(i)}\left(d_{*}\right)-R_{b}^{(i)}\left(d_{*}\right)$ reduces to consideration of $Z_{a}^{(l)}-Z_{b}^{(l)}, l=0, \ldots, i$. Now note from (9) that for all $a, b, C_{a}^{\prime} C_{b}=2 \pi G_{a b}$. Then from (26) it follows that $\left\|C_{a}\right\|^{2}=\left\|C_{b}\right\|^{2}=\left|C_{a}^{\prime} C_{b}\right|$, with $\|$.$\| denoting Eucliean norm, to imply,$ by the Schwarz inequality, that $C_{a}= \pm C_{b}$, so that $Z_{a}^{(l)}-Z_{b}^{(l)}$ is identically zero. Thus the first term in $(25)$ is $O_{p}\left((\log m)(m / n)^{\xi}+(\log m)^{2} m^{-2 / 3}\right)$. Since $R_{b}^{(1)}\left(d_{*}\right)=O_{p}\left(m^{-1 / 2}\right)$, the second term in $(25)$ is $O_{p}\left((\log m)^{2}(m / n)^{\xi} m^{-1 / 2}+\right.$ $\left.(\log m)^{2} m^{-7 / 6}\right)$, and the proof is completed.

Proof of Proposition 2 We give only partial details as much of the proof is similar to that of Theorem 2 of Robinson (1995b) and the Theorem of Lobato (1999). We have

$$
m^{1 / 2}\left\{\hat{G}\left(d_{*}\right)-G\right\}=\frac{1}{m^{1 / 2}} \sum_{j=1}^{m} \lambda_{j}^{2 d_{*}}\left(\operatorname{Re}\left(I_{j}\right)-G\right)=\eta+\zeta
$$


where

$$
\begin{aligned}
\eta & =m^{-1 / 2} \sum_{j=1}^{m}\left\{\lambda_{j}^{2 d_{*}}\left(\operatorname{Re}\left(I_{j}-A_{j} J_{j} A_{j}^{*}\right)\right)+\left(\lambda_{j}^{2 d} f\left(\lambda_{j}\right)-G\right)\right\} \\
\zeta & =m^{-1 / 2} \sum_{j=1}^{m} \operatorname{Re}\left(B_{j}\left(J_{j}-I_{p} / 2 \pi\right) B_{j}^{*}\right)=\frac{m^{-1 / 2}}{2} \sum_{j}^{\prime} B_{j}\left(J_{j}-I_{p} / 2 \pi\right) B_{j}^{*}
\end{aligned}
$$

where $\sum_{j}{ }^{\prime}=\sum_{j=1}^{m}+\sum_{j=n-m}^{n-1}$ and we write $A_{j}=A\left(e^{i \lambda_{j}}\right), B_{j}=\lambda_{j}^{d} A_{j}, 1 \leq$ $j \leq m, B_{j}=\lambda_{n-j}^{d} A_{j}, n-m \leq j \leq n-1$, and use the fact that $\lambda_{j}^{2 d_{*}} f\left(\lambda_{j}\right)=$ $B_{j} B_{j}^{*} / 2 \pi, 1 \leq j \leq m$.

Appendices C and D of Lobato (1999) assure under Assumptions A, C and D that $\eta=o_{p}(1)$. Now $\zeta=\zeta_{1}+\zeta_{2}$, where

$$
\begin{aligned}
\zeta_{1} & =\frac{m^{-1 / 2}}{2} \sum_{j}^{\prime} B_{j}\left(\frac{1}{2 \pi n} \sum_{t=1}^{n}\left(e_{t} e_{t}^{\prime}-I_{p}\right)\right) B_{j}^{*}, \\
\zeta_{2} & =\frac{m^{-1 / 2}}{2} \sum_{j}^{\prime} B_{j}\left(\frac{1}{2 \pi n} \sum_{t=1}^{n} \sum_{s=1, s \neq t}^{n} e_{t} e_{s}^{\prime} e^{-i(t-s) \lambda_{j}}\right) B_{j}^{*}
\end{aligned}
$$

Because $\sum_{t=1}^{n}\left(e_{t} e_{t}^{\prime}-I_{p}\right)=O_{p}\left(n^{1 / 2}\right)$, we have $\zeta_{1}=O_{p}\left((m / n)^{1 / 2}\right)=o_{p}(1)$. By the Cramer-Wold device it remains to show that for any non-null $p^{2} \times 1$ vector $\tau$,

$$
\tau^{\prime} \operatorname{vec}\left(\zeta_{2}\right) \rightarrow_{d} N\left(0, \frac{1}{2} \tau^{\prime}\left\{G \otimes G+\left(G \otimes G_{1}, \ldots, G \otimes G_{p}\right\} \tau\right)\right.
$$

Now, writing $H_{j}=\bar{B}_{j} \otimes B_{j}, \operatorname{vec}\left(\zeta_{2}\right)$ is $m^{-1 / 2} / 4 \pi n$ times

$$
\begin{aligned}
\sum_{t=1}^{n} \sum_{s=1, s \neq t}^{n} \sum_{j}^{\prime} H_{j} e^{i(s-t) \lambda_{j}}\left(e_{s} \otimes e_{t}\right) & =\sum_{t=1}^{n} \sum_{s=1, s<t}^{n}\left(C_{s t}\left(e_{s} \otimes e_{t}\right)+C_{t s}\left(e_{t} \otimes e_{s}\right)\right) \\
& =\sum_{t=1}^{n}\left\{\sum_{s=1, s<t}^{n}\left(C_{s t}+C_{t s} P\right)\left(e_{s} \otimes I_{p}\right)\right\} e_{t},
\end{aligned}
$$

where $C_{s t}=\sum_{j}{ }_{j} H_{j} e^{i(s-t) \lambda_{j}}$ and $P$ is the $p^{2} \times p^{2}$ permutation matrix such that $x \otimes y=P(y \otimes x)$ for all $p \times 1$ vectors $x, y$. Thus $\tau^{\prime} \operatorname{vec}\left(\zeta_{2}\right)=\sum_{t=1}^{n} Z_{t}$, where, 
with $U_{t}=\frac{m^{-1 / 2}}{4 \pi n} \tau^{\prime}\left(\sum_{s=1, s<t}^{n}\left(C_{s t}+C_{t s} P\right)\left(e_{s} \otimes I_{p}\right)\right), \quad Z_{t}=U_{t} e_{t}$ is a martingale difference with respect to $\mathcal{F}_{t}$. Put $V_{n}^{2}=\sum_{t=1}^{n} E\left\{Z_{t}^{2} \mid \mathcal{F}_{t-1}\right\}$, where $\mathcal{F}_{0}$ is the trivial $\sigma$-field $\{\phi, \Omega\}$ and $s_{n}^{2}=\sum_{t=1}^{n} E\left(Z_{t}\right)^{2}$. By the same argument as in the proof of Theorem 2 of Robinson (1995b), and in Appendix A of Lobato (1999), as $n \rightarrow \infty V_{n}^{2} s_{n}^{-2} \longrightarrow p 1$ and, for any $\epsilon>0, s_{n}^{-2} \sum_{t=1}^{n} E\left\{Z_{t}^{2} 1\left(\left|Z_{t}\right| \geq\right.\right.$ $\left.\left.\epsilon s_{n}\right)\right\} \rightarrow 0$. Hence by Theorem 2 of Brown (1971), $\sum_{t=1}^{n} Z_{t} / s_{n} \longrightarrow{ }_{d} N(0,1)$. It thus remains to show that $s_{n} \rightarrow \frac{1}{2} \tau^{\prime}\left\{G \otimes G+\left(G \otimes G_{1}, \ldots, G \otimes G_{p}\right)\right\} \tau$, which is easily seen to reduce to showing that

$\frac{1}{m n^{2}} \sum_{t=1}^{n} \sum_{s=1, s<t}^{n}\left(C_{s t}+C_{t s} P\right)\left(C_{s t}^{\prime}+P^{\prime} C_{t s}^{\prime}\right) \rightarrow 8 \pi^{2}\left\{G \otimes G+\left(G \otimes G_{1}, \ldots, G \otimes G_{p}\right)\right\}$.

The left side is

$$
\begin{aligned}
& \frac{1}{2 m n^{2}} \sum_{t=1}^{n} \sum_{s=1, s \neq t}^{n} \sum_{j}^{\prime} H_{j}\left(e^{i(s-t) \lambda_{j}}+e^{i(t-s) \lambda_{j}} P\right) \sum_{k}^{\prime}\left(e^{i(t-s) \lambda_{k}}+e^{i(s-t) \lambda_{k}} P\right) H_{k}^{*} \\
= & \frac{1}{m} \sum_{j}^{\prime} H_{j}\left(H_{j}^{*}+P H_{n-j}^{*}\right)-\frac{1}{2 m n} \sum_{j}^{\prime} H_{j}\left(I_{p^{2}}+P\right) \sum_{k}^{\prime}\left(I_{p^{2}}+P\right) H_{k}^{*} .
\end{aligned}
$$

The second term is easily seen to be $O_{p}(m / n)$. The first term can be written

$$
\frac{1}{m} \sum_{j}^{\prime}\left\{\left(\bar{B}_{j} B_{j}^{\prime} \otimes B_{j} B_{j}^{*}\right)+\left(\bar{B}_{j} \otimes B_{j}\right)\left(B_{j}^{\prime} \otimes B_{1 j}^{*}, \ldots, B_{j}^{\prime} \otimes B_{p j}^{*}\right)\right\}
$$

where $B_{i j}^{*}$ is the $i$ th column of $B_{j}^{*}$. Since $B\left(e^{i \lambda}\right) B\left(e^{i \lambda}\right)^{*} / 2 \pi \rightarrow G$ as $\lambda \rightarrow 0$, and $G$ is symmetric, the proof is readily completed.

Proof of Proposition 3 From Proposition 2 it suffices to show that $\widehat{G}\left(\bar{d}_{*}\right)-\widehat{G}\left(d_{*}\right)=o_{p}\left(m^{-1 / 2}\right)$. From Proposition 1 we have $\bar{d}_{*}-d_{*}=O_{p}\left(m_{1}^{-1 / 2}\right)$.

We thus have, for any $\varepsilon>0, \eta>0$

$$
\begin{aligned}
& P\left\{m^{1 / 2}\left\|\widehat{G}\left(\bar{d}_{*}\right)-\widehat{G}\left(d_{*}\right)\right\|>\epsilon\right\} \leq P\left\{m^{1 / 2}\left\|\widehat{G}\left(\bar{d}_{*}\right)-\widehat{G}\left(d_{*}\right)\right\|>\epsilon,\left|\bar{d}_{*}-d_{*}\right| \leq \eta\right\} \\
& +o(1) \text {, }
\end{aligned}
$$


as $n \rightarrow \infty$. By the mean value theorem, for small enough $\eta$ and $\left|\bar{d}_{*}-d_{*}\right| \leq \eta$, $\left\|\widehat{G}\left(\bar{d}_{*}\right)-\widehat{G}\left(d_{*}\right)\right\|$ is bounded by

$$
\frac{2 \log n}{m}\left|\bar{d}_{*}-d_{*}\right| \sum_{j=1}^{m} \lambda_{j}^{2\left(d_{*}-\eta\right)} \operatorname{tr}\left\{I_{j}\right\}=O_{p}\left(\frac{\log n}{m}\left|\bar{d}_{*}-d_{*}\right| \sum_{j=1}^{m} \lambda_{j}^{-2 \eta}\right)
$$

due to $E I_{a a}\left(\lambda_{j}\right)=O\left(\lambda_{j}^{-2 d}\right), a=1, \ldots, p$ [see Robinson (1995a, Theorem 2)]. The right side is $O_{p}\left(m_{1}^{-1 / 2}(n / m)^{2 \eta} \log n\right)=O_{p}\left(m_{1}^{-1 / 2}(n / m)^{\zeta}\right)$ for $\zeta>2 \eta$. From Assumption $\mathrm{H}$, this is $o_{p}\left(m^{-1 / 2}\right)$, to complete the proof.

Proof of Proposition 4 Let $Q$ be a $p \times p$ orthogonal matrix such that $Q G Q^{\prime}=\Delta=\operatorname{diag}\left\{\delta_{1}, \ldots, \delta_{p}\right\}$. Then the eigenvalues of $\hat{G}\left(\bar{d}_{*}\right)$ are identical with those of $Q \hat{G}\left(\bar{d}_{*}\right) Q^{\prime}$. Put $U(n)=m^{1 / 2}\left(Q \hat{G}\left(\bar{d}_{*}\right) Q^{\prime}-\Delta\right)$. From Proposition 3 it readily follows that

$$
v e c\{U(n)\} \rightarrow{ }_{d} N\left(0, \frac{1}{2}\left\{\Delta \otimes \Delta+\left(\Delta \otimes \Delta_{1}, \ldots, \Delta \otimes \Delta_{p}\right)\right\}\right)
$$

where $\Delta_{i}$ is the $i t h$ column of $\Delta$. The proof can then be completed by the argument of Theorem 1 of Anderson (1963) and Theorem 13.5.1 of Anderson (1984), noting that the limit distribution in Proposition 3 is the same as that of $m^{1 / 2} \operatorname{vec}\left\{W_{p}(2 m, G) /(2 m)-G\right\}$ as $m \rightarrow \infty, W_{p}$ denoting a Wishart variate, and, on p.141 of Anderson (1963), replacing $r$ by $p-r+1$, taking $q_{i}=1, q_{p-r+1}=r$, and then putting $\lambda_{i}=\delta_{i}, i=1, \ldots, p-r, \lambda_{p-r+1}=0$.

Proof of Theorem 3. A straightforward application of Proposition 4 and the delta method.

Proof of Theorem 4. We have

$$
P(\widehat{r}>r) \leq \sum_{u=r+1}^{p-1} P(L(u)<L(r)) \leq p P\left(\widehat{\delta}_{p-r}<p v(n)\right) \rightarrow 0
$$

as $n \rightarrow \infty$ since $\widehat{\delta}_{p-r} \rightarrow{ }_{p} \delta_{p-r}>0$ and $v(n) \rightarrow 0$ by Proposition 4 and 
Assumption J. On the other hand

$$
P(\widehat{r}<r) \leq \sum_{u=1}^{r-1} P(L(u)<L(r)) \leq r P\left(m^{1 / 2} \widehat{\delta}_{p-r+1}>m^{1 / 2} v(n)\right) \rightarrow 0
$$

as $n \rightarrow \infty$ since $\widehat{\delta}_{p-r+1} \rightarrow_{p} 0$ and $m^{1 / 2} v(n) \rightarrow \infty$ by Proposition 4 and Assumption J. The proof is complete.

\section{References}

Abadir, K.M., Taylor, A.M.R., 1999. On the definition of (co-)integration. Journal of Time Series Analysis 20, 129-137.

Adenstedt, R.K., 1974. On large-sample estimation for the mean of a stationary random sequence. Annals of Statistics 2,1095-1107.

Anderson, T.W., 1963. Asymptotic theory for principal component analysis. Annals of Mathematical Statistics 34, 122-148.

Anderson, T.W., 1984. An Introduction To Multivariate Statistical Analysis. 2nd edition. Wiley, New York. 
Banerjee, A., Dolado, J., Galbraith, J.W., Hendry, D.F., 1993. Cointegration, Error Correction, and the Econometric Analysis of Non-Stationary Data. Oxford University Press, Oxford.

Brillinger, D.R., 1975. Time Series, Data Analysis and Theory. Holt, Rinehart and Winston, New York.

Brown, B.M., 1971. Martingale central limit theorems. Annals of Mathematical Statistics 42, 59-66.

Cheung, Y-W., Lai, K.S., 1993. A fractional cointegration analysis of purchasing power parity. Journal of Business and Economic Statistics 11, 103-112.

Dickey, D.A., Fuller, W.A., 1981. Likelihood ratio statistics for autoregressive time series with a unit root. Econometrica 49, 1057-1072.

Diebold, F.X., Rudebusch, G.D., 1991. On the power of Dickey-Fuller tests against fractional alternatives. Economic Letters 35, 155-160.

Engle, R. F., Granger, C.W.J., 1987. Co-integration and error correction: representation, estimation and testing. Econometrica 55, 251-276.

Engle, R. F., Granger, C.W.J., 1991. Long-Run Economic Relationships. Oxford University Press, Oxford.

Flôres Jr., R.G., Szafarz, A., 1996. An enlarged definition of cointegration. Economics Letters 50, 193-195.

Fujikoshi, Y., 1985. Two methods for estimation of dimensionality in canonical correlation analysis and the multivariate linear model. In K.Matusita, (Ed.) Statistical Theory and Data Analysis, pp 233-240. Elsevier, Amsterdam.

Fujikoshi, Y., Veitch, L.G., 1979. Estimation of dimensionality in canonical correlation analysis. Biometrika 66, 345-351. 
Geweke, J., Porter-Hudak, S., 1983. The estimation and application of long memory time series models. Journal of Time Series Analysis 4, 221-238.

Gunderson, B.K., Muirhead, R.J., 1997. On estimating the dimensionality in canonical correlation analysis. Journal of Multivariate Analysis 62, $121-136$

Hatanaka, M., 1996. Time-Series-Based Econometrics: Unit Roots and Cointegration. Oxford University Press, Oxford.

Henry, M., Robinson, P.M., 1996. Bandwidth choice in Gaussian semiparametric estimation of long-range dependence. In Robinson, P.M. and Rosenblatt, M. (Eds.) Athens Conference on Applied Probability and Time Series Analysis, pp.220-232. Springer-Verlag, New York.

Hsu, J.C., 1996. Multiple Comparisons: Theory and Methods. Chapman and Hall, London.

Johansen, S., 1996. Likelihood-Based Inference In Cointegrated Vector Autoregressive Models. 2nd Printing. Oxford University Press, Oxford.

Künsch, H., 1987. Statistical aspects of self-similar processes. In Prohorov, $\mathrm{Yu}$ and Sazonov, V.V. (Eds.) Proceedings of the First World Congress of the Bernoulli Society 1, pp 67-74. VNU Science Press, Utrecht.

Liu, C.L., 1968. Introduction to Combinatorial Mathematics. McGrawHill, New York.

Lobato, I., 1995. Multivariate analysis of long memory series in the frequency domain. Ph.D. Thesis. London School of Economics.

Lobato, I., 1999. A semiparametric two-step estimator in a multivariate long memory model. Journal of Econometrics 90, 129-153.

Marcus, R., Peritz, E., Gabriel, K.R.,1976. On closed testing procedures with special references to ordered analysis of variance. Biometrika 63, 655- 
660.

MacKinnon, J., 1991. Critical values for cointegration tests. In Engle, R.F and Granger, C.W.J. (Eds.) Long-Run Economic Relationships, pp. 267-276. Oxford University Press, Oxford.

Phillips, P.C.B., Ouliaris, S., 1988. Testing for cointegration using principal components methods. Journal of Economic Dynamics and Control 12, 205-230

Phillips, P.C.B., Ouliaris, S., 1990. Asymptotic properties of residual based cointegration tests. Econometrica 58, 165-193.

Robinson, P.M., 1994. Semiparametric analysis of long-memory time series. Annals of Statistics 22, 515-539.

Robinson, P.M., 1995a. Log-periodogram regression of time series with long range dependence. Annals of Statistics 23, 1048-1072.

Robinson, P.M., 1995b. Gaussian semiparametric estimation of long range dependence. Annals of Statistics 23, 1630-1661.

Robinson, P.M., Marinucci, D., 1998. Semiparametric frequency domain analysis of fractional cointegration. STICERD Econometrics Discussion Paper EM/98/348, London School of Economics.

Said, S.E., Dickey, D.A., 1984. Testing for unit roots in autoregressivemoving average models of unknown order. Biometrika 71, 599-607.

Stock, J.H., Watson, M., 1988. Testing common trends. Journal of the American Statistical Association 83, 1097-1107.

Velasco, C., 1999. Gaussian semiparametric estimation of nonstationary time series. Journal of Time Series Analysis 20, 87-127.

Yong, C.H., 1974. Asymptotic Behaviour of Trigonometric Series. Chinese University of Hong Kong, Hong Kong. 
Table 1: ADF test

\begin{tabular}{lccc}
\hline Series & $t$-ratio & $5 \%$ critical value & $1 \%$ critical value \\
\hline WTI & -3.489 & -2.883 & -3.480 \\
BRENT & -3.019 & -2.883 & -3.479 \\
DUBAI & -4.691 & -2.882 & -3.477 \\
\hline
\end{tabular}


Table 2: Estimates $\widehat{d}_{a}$ of $d_{a}, a=1,2,3$, with standard errors in parentheses.

\begin{tabular}{lcc}
\hline Series & $m=20$ & $m=15$ \\
\hline WTI & $0.5336(0.112)$ & $0.4714(0.129)$ \\
BRENT & $0.4538(0.112)$ & $0.3748(0.129)$ \\
DUBAI & $0.4367(0.112)$ & $0.3076(0.129)$ \\
\hline
\end{tabular}


Table 3: Estimates $\widehat{G}$ of $G, m=13$

\begin{tabular}{lccc}
\hline Series & WTI & BRENT & DUBAI \\
\hline WTI & 0.0037456 & 0.0046078 & 0.0055116 \\
BRENT & 0.0046078 & 0.0064323 & 0.0073090 \\
DUBAI & 0.0055116 & 0.0073090 & 0.0090621 \\
\hline
\end{tabular}


Table 4: Estimates $\widehat{G}\left(\bar{d}_{*}\right)$ of $G$ based on $\bar{d}_{*}=\left(\widehat{d}_{1}+\widehat{d}_{2}+\widehat{d}_{3}\right) / 3$, and eigenvalues of $\widehat{G}\left(\bar{d}_{*}\right), \widehat{P}\left(\bar{d}_{*}\right) ; m=13, m_{1}=15$

\begin{tabular}{lccc}
\hline Series & WTI & BRENT & DUBAI \\
\hline WTI & 0.0049261 & 0.0054196 & 0.0057460 \\
BRENT & 0.0054196 & 0.0062486 & 0.0065317 \\
DUBAI & 0.0057460 & 0.0065317 & 0.0072951 \\
\hline eigenvalues & 1 & 2 & 3 \\
\hline$G\left(\times 10^{-2}\right)$ & 1.80704 & 0.02750 & 0.01244 \\
$\mathrm{P}$ & 2.93521 & 0.04298 & 0.02182 \\
\hline
\end{tabular}


Table 5: Estimates $\widehat{G}_{(1)}\left(\bar{d}_{*}\right)$ of $G_{(1)}$ (Brent and Dubai only) based on

$\bar{d}_{*}=\left(\widehat{d}_{1}+\widehat{d}_{2}\right) / 2$, and eigenvalues of $\widehat{G}_{(1)}\left(\bar{d}_{*}\right)$,
\begin{tabular}{lcc}
\multicolumn{3}{c}{$m=13, m_{1}=15$} \\
\hline Series & BRENT & DUBAI \\
\hline BRENT & 0.0071133 & 0.0073984 \\
DUBAI & 0.0073984 & 0.0082350 \\
\hline eigenvalues & 1 & 2 \\
\hline$G\left(\times 10^{-2}\right)$ & 1.50938 & 0.02545 \\
$\mathrm{P}$ & 1.96665 & 0.03335 \\
\hline
\end{tabular}

